\title{
Perspectivas metodológicas para o estudo da gestão da informação em ambientes informacionais das organizações
}

\author{
Methodological perspectivas for the study of information management in organizational environments
}

\author{
Cássia Regina Bassan de Moraes (1) y Bárbara FADEL (2)
}

(1) Faculdade de Filosofia e Ciências, Unesp, Av. Hygino Muzzi Filho, 737, Marília, São Paulo, Brasil CEP 17525-900, cassiabassan@flash.tv.br (2) Faculdade de Filosofia e Ciências, Unesp e Centro Universitário de Franca, Uni-FACEF, Av. Major Nicácio, 2433, Franca, São Paulo, Brasil, CEP 14.401-135, bafadel@terra.com.br.

\begin{abstract}
Resumen
Las investigaciones sobre el comportamiento de las personas en la utilización de las Tecnologías de Información y Comunicación (TICs) en las organizaciones para la gestión de la información vienen siendo criticadas por predominar en ellas puntos de vista positivistas y por el uso intenso de metodologías cuantitativas. En ese sentido, la propuesta del presente estudio es la de abordar el método de la triangulación como perspectiva para la investigación de la complejidad de la gestión de la información en las organizaciones. La triangulación es un paso dado por los investigadores para integrar interpretaciones de datos diversas procedentes de entornos y momentos diferentes. En este sentido, la perspectiva de utilización de la metodología de la triangulación busca integrar varios métodos, con el objetivo de obtener una mejor percepción de fenómenos que normalmente no son visualizados con los métodos existentes aplicados de forma aislada. La metodología citada permite utilizar varias herramientas con miras a colectar tanto datos objetivos (cuantitativos) como subjetivos (cualitativos), de forma que se obtenga al final de la colecta un panorama completo de las vivencias cotidianas en el ambiente informacional de las organizaciones. De esta forma, se demuestra la existencia de un campo de estudio que puede contribuir al desarrollo de la investigación en la gestión de la información.
\end{abstract}

Palabras clave: Gestión de la información. Investigación. Metodología. Epistemología. Triangulación. Ambiente informacional.

\section{Ambientes informacionais e as TIC's}

No estudo dos ambientes informacionais, podese considerar um aspecto micro e um aspecto macro. No primeiro aspecto, são levados em conta os aspectos internos das organizações; já para o segundo, consideram-se os aspectos internos e também os externos

\begin{abstract}
Studies on the behavior of people when using Information and Communication Technologies (ICT) for information management in their organizations are being criticized for their predominating positivists points of view and for the intense use of quantitative methodologies. This study proposes the method of triangulation as a perspective for the inquiry of the complexity involved in the study of information management in organizations. The triangulation is a step took by the researchers to integrate some diverse data interpretations from different times and environments. In this way, triangulation pretends to integrate different methods, with the objective of getting a better perception of phenomena that are not normally perceived by the existing methods when applied in isolation. The metholodogy allows for the integration of qualitative and quantitative approaches and data, contribuiting to the existing research models in information management.
\end{abstract}

Keywords: Information Management. Research. Methodology. Epistemology. Triangulation. Informational environment.

Considerando os aspectos micro, Valentim (2002) afirma que as organizações são formadas por três diferentes ambientes:

\footnotetext{
- O primeiro está ligado ao próprio organograma, isto é, as inter-relações entre as diferentes unidades de trabalho como diretorias, gerências, divisões, departamentos, setores, seções etc.;
} 
- O segundo está relacionado à estrutura de recursos humanos, isto é, as relações entre pessoas das diferentes unidades de trabalho e,

- O terceiro e último, é composto pela estrutura informacional, ou seja, geração de dados, informação e conhecimento pelos dois ambientes anteriores.

Ainda segundo a autora, a partir do reconhecimento desses três ambientes, pode-se mapear os fluxos informais de informação existentes na organização, assim como se pode estabelecer fluxos formais de informação para consumo da própria organização, conforme ilustra a figura a seguir:

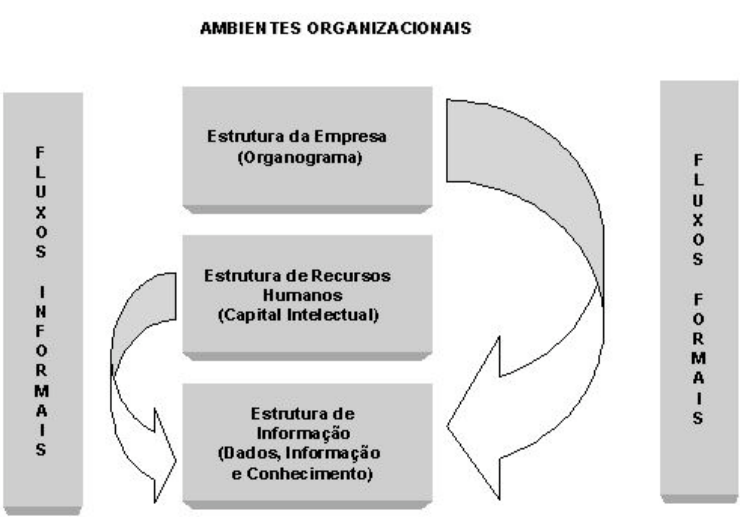

Figura 1

[Fonte: Valentim - 2002]

Para Moraes e Fadel (2006, p. 102), pensando num aspecto macro, devem-se considerar três ambientes para o estudo da informação: a) o ambiente externo (negócios, informação, tecnologia); b) o ambiente organizacional (negócios, espaço físico, tecnologia); e c) o ambiente informacional.

Segundo Davenport e Prusak (2001, p. 50), o ambiente informacional está arraigado no ambiente mais amplo que o envolve, o organizacional, e ambos são afetados pelo ambiente externo, o mercado. Na prática, esses ambientes se sobrepõem e têm limites indistintos.

Sem dúvida, quando se fala em ambiente externo, fala-se do mundo capitalista. Uma das principais características deste mundo é a capacidade de atender às novas exigências do mercado e, desse modo, observa-se que o ambiente informacional das organizações se modifica constantemente, uma vez que a natureza da informação é dinâmica (Moraes e Fadel, p. 99).

Segundo Pochmann (2003), não sem motivo, o avanço da fronteira tecnológica transforma-se recorrentemente na forma de potencializar o processo de acumulação de capital e de eliminação dos concorrentes.

Muito embora a inovação técnica tenha presença constante ao longo do desenvolvimento econômico, pode-se observar que certos momentos históricos concentram um conjunto de modificações tecnológicas, com capacidade de alterar radicalmente não apenas o processo produtivo, mas também a conformação de toda uma sociedade.

A rápida difusão de uma nova onda de inovação não só modifica a base técnica responsável pela dinâmica do ciclo de acumulação de capital, mas também termina por influenciar os mais distintos processos de produção e de trabalho, a partir do aumento dos lucros, dos ganhos de produtividade e da queda dos preços, com destaque para os segmentos modernos e mais dinâmicos.

Em relação aos três últimos séculos, houve pelo menos duas grandes ondas de profundas inovações, que podem ser chamadas de revolução tecnológica (Pochmann, 2003) ou de Revolução Industrial (Castells, 2001). Ambos os autores concordam com o fato de que tanto a primeira onda de inovação, iniciada pouco antes dos últimos trinta anos do século XVIII, como a segunda onda de inovação, ocorrida cerca de cem anos depois, foram marcas constitutivas da profunda modificação nas bases técnica e material do capitalismo contemporâneo, capaz de assegurar novos ciclos de acumulação de capital.

$\mathrm{Na}$ primeira onda de inovação, as atividades econômicas não vinculadas à produção de alimentos foram o núcleo dinâmico do processo de industrialização e que proporcionaram à Inglaterra o exercício da hegemonia no cenário internacional. Em grande medida, isso foi possibilitado pela onda de inovação concentrada naquele país.

$\mathrm{Na}$ segunda revolução tecnológica, pode-se constatar uma radical modificação na divisão do trabalho, o que coincidiu justamente com a descoberta de novos materiais, como o aço e o petróleo, a energia elétrica, o motor a combustão, o telégrafo, o telefone, entre outros.

O capitalismo passa por um novo período de aprofundamento nas descobertas técnicas e científicas nas duas últimas décadas do século $X X$. As inovações nos campos da informática, telemática, novos materiais, e biotecnologia impulsionam a transformação do padrão de organização da produção e do trabalho nas mais diversas atividades econômicas. 
Segundo Pochmann (2003), diante das novas possibilidades constituídas e de suas perspectivas, alguns autores têm procurado tratar do conceito de terceira revolução tecnológica na forma de distintos entendimentos, tais como: revolução da informática (Harvey, 1992; Coriat, 1988), sociedade informática e/ou da informação (Schaff, 1995; Lojkine, 1995), a sociedade do tempo livre e/ou a sociedade do conhecimento (Masi, 1999), a sociedade pós-industrial (Bell, 1973; Gorz, 1994) e a economia em rede (Castells, 2001).

Em grande medida, registra-se a presença de uma verdadeira convergência desta terceira onda de inovação nos meios de comunicação, capaz de alterar profundamente os modos de produção, de trabalho e de vida. O aparecimento e desenvolvimento do computador e a sua mais recente associação junto aos meios de comunicação já existentes, como a televisão e o telefone, confirmam a passagem para um estágio superior na produção de informações e comunicações.

Em relação ao computador, que ao final do século XX constitui uma tecnologia mais recente de comunicação, nota-se que desde os anos 1960 as modificações no computador tem sido amplas.

Além da ampliação da capacidade de processamento do computador, a sua miniaturização o tornou um bem de consumo durável cada vez mais massificado. Assim, o acesso ao computador através de seu uso em rede (Internet) possibilitou um novo salto nas comunicações.

As inovações tecnológicas não trilham caminhos separados: com a possível convergência entre as três principais tecnologias de comunicação (telefone, televisão e computador), potencializase um novo estágio em termos das comunicações minimizando o poder da geografia através da redução da distância.

A atual revolução tecnológica caracteriza-se não pela centralidade de conhecimentos e informação, mas pela aplicação desses conhecimentos e dessa informação para a geração de novos conhecimentos e de dispositivos de processamento/comunicação da informação, em um ciclo de realimentação cumulativo entre a inovação e seu uso.

Portanto, segundo Moraes e Fadel (2006, p. 103), a nova sociedade emergente desse processo de transformação é capitalista e também informacional, embora apresente variação histórica considerável nos diferentes países, conforme sua história, cultura, instituições e relação específica com o capitalismo global e a tecnologia informacional.

A revolução tecnológica atual vem causando uma mudança no cenário competitivo das organizações. De acordo com Fleury (2003), com o objetivo de alcançarem maiores índices de competitividade, as organizações têm utilizado variada e complexa gama de tecnologias. Por isso, novas tecnologias podem ser encontradas em vários ambientes, com reflexos diferentes em cada um deles, em virtude das peculiaridades inerentes a cada contexto. Desde o planejamento de novos produtos, da reorganização de processos produtivos, passando pela adoção de novos modelos de gestão administrativa, as novas tecnologias têm sido adotadas como ataIhos para o alcance de melhores resultados.

Esse fato vem exigindo rápidas e contínuas adaptações na postura estratégica dessas organizações, para sobreviver e crescer. A mudança tecnológica acaba tendo um forte impacto psicológico e sociológico, pois obriga as pessoas a pensar novas maneiras de gerenciamento, bem como novos padrões de eficiência e produtividade.

Os gestores buscam investir em novas tecnologias de informações partindo da crença que a corporação do futuro será uma empresa altamente computadorizada, e a sua competitividade e sobrevivência dependerão de como ela usará a automação, segundo Martin (1991); utilizam-nas para ações estratégicas e para planejar e alcançar uma ou mais das três funções independentes: a) aumentar a continuidade (integração funcional, automação intensificada, resposta rápida); b) melhorar o controle (precisão, acuidade, previsibilidade, consistência, certeza); e c) proporcionar maior compreensão (visibilidade, análise, síntese) das funções produtivas.

Atualmente, as organizações bem sucedidas, ou seja, aquelas que têm conseguido atingir os seus objetivos estratégicos possuem as seguintes características: são ágeis nas decisões; inovadoras nos processos, produtos e serviços; enfatizam o binômio produtividade e qualidade; preocupam-se com a evolução tecnológica e com a preservação ambiental; reconhecem o alto valor estratégico dos Sistemas de Informação, investindo na sua disseminação e utilização.

\section{A gestão da informação}

As organizações, cada vez mais, se dão conta de que a informação é um recurso estratégico que tem custo, preço e valor. Sendo assim, 
existe a necessidade dela ser gerenciada assim como são os recursos financeiros, materiais e humanos. Essa crescente necessidade de gerenciar a informação, levando-se em conta também os aspectos humanos e das tecnologias de informação e comunicação, teve como resultado a proposta da formação de uma área profissional, originalmente chamada de Information Resources Management (Moraes e Fadel, 2006, p. 107).

Traduzida como Gerenciamento de Recursos Informacionais, e atualmente mais conhecida como Gestão da Informação, esta nova área se configura como um campo de estudos já consolidado nos Estados Unidos e na Europa, cujos conteúdos teóricos e operacionais têm se transformado em ferramenta imprescindível para qualquer organização que necessite produzir, localizar, coletar, tratar, armazenar, distribuir e estimular a geração e o uso da informação. Segundo Ponjuán Dante (2007, p. 19):

La gestión es un proceso, por lo que se definiría la gestión de información como 'el proceso mediante el cual se obtienen, despliegan o utilizan recursos básicos (económicos, físicos, humanos, materiales,) para manejar información dentro y para la sociedad a la que sirve. Tiene como elemento básico la gestión del ciclo de vida de este recurso y ocurre en cualquier organización. Es propia también de unidades especializadas que manejan este recurso en forma intensiva, llamadas unidades de información'. El proceso de gestión de información debe ser valorado sistémicamente en diferentes dimensiones y el dominio de sus esencias permite su aplicación en cualquier organización.

Ainda segundo a autora, os fluxos que ocorrem em todo o sistema devem ser objeto de gestão. Estes processos respondem a cada organização, porque tem características particulares. Entretanto, em geral podem associar a segmentos típicos da organização ou a uma dimensão, de tal forma que se podem identificar as seguintes dimensões: o ambiente, os processos, as pessoas, a tecnologia, a infra-estrutura, e os produtos e os serviços.

Estas dimensões possuem uma estreita relação, e podem ser abordadas de diferentes formas.

Para se fazer a Gestão da informação de uma organização há a necessidade de: um domínio dos diferentes tipos de informações que se manejam na organização; a dinâmica de seus fluxos (representados em diversos processos que transitam em cada informação);o ciclo de vida de cada informação (incluída a gestão de geração da informação, onde quer que ela ocorra); e o conhecimento das pessoas sobre o manejo da informação e da sua cultura informacional.
Porém, dificilmente uma organização alcançará a vantagem competitiva, sem utilizar abordagens e métodos de gestão mais voltados às pessoas, muito menos sem propiciar a interação com a informação e com o conhecimento.

Sobre a questão da gestão, via sistemas de informação, e as pessoas, Ponjuán Dante (2007, p. 91) faz as seguintes considerações:

En los sistemas de información, algunas operaciones de mayor o menor complejidad pueden ser efectuadas por maquinas, pero estas han sido diseñadas y son manejadas por personas. Son los seres humanos los que se responsabilizan de la calidad, los que pueden marcar las diferencias entre procesos $u$ organizaciones y aun entre ellos mismos. Una de las principales entidades de los sistemas de información es el hombre. De hecho, en la conformación de los sistemas de información hay una notable presencia de personas. Podrían no existir equipos $u$ otros medios, pero el hombre es el que genera, analiza, filtra, interpreta, integra y usa información. De personas de diferente nivel, de diversas especialidades, depende el éxito de los sistemas de información.

Para tanto, as organizações necessitam tratar seus colaboradores como sujeitos na sua totalidade e complexidade, cujas potencialidades precisam ser incentivadas e valoradas, uma vez que todas as pessoas da organização participam da cadeia informacional e comunicacional, uma vez que são as pessoas que se vinculam com os usuários e com os provedores, e são elas que tomam decisões, favoráveis ou não; também são elas que desenham os produtos e serviços interpretando as necessidades dos usuários.

Assim a gestão da informação possui papel fundamental porque propicia a melhoria dos fluxos informacionais, agregando dinamicidade, valor e controle, através de métodos, técnicas, procedimentos e ferramentas de gestão que otimizam o desempenho da organização, mas sempre com o foco nas pessoas que participam do processo.

Segundo Moraes e Fadel (2006, p. 101), Davenport e Prusak criaram a metáfora Ecologia da Informação, para sugerir uma nova maneira de gerenciar a informação. Para o autor, a escolha do termo ecologia não se refere, como alguns chegaram a pensar, a uma administração verde, mas sim à ciência de compreender e administrar todos os ambientes. Em outras palavras, a ecologia da informação seria a "administração holística da informação, ou a administração informacional centrada no ser humano". (Davenport e Prusak, 2001, p. 44). A ecologia da informação, além de exigir um modelo holístico de pensar, tem quatro atributos que Daven- 
port e Prusak consideram essenciais: integração dos diversos tipos de informação; reconhecimento de mudanças evolutivas; ênfase na observação e descrição; e ênfase no comportamento pessoal e informacional.

Como se pode perceber, diferentes acepções sobre gestão da informação perpassam a questão das pessoas.

Desta forma, quando se pensa em uma metodologia para o estudo da gestão da informação, há que se levar em consideração métodos que envolvam não apenas aspectos mensuráveis, mas, principalmente, os que investigam os aspectos subjetivos e perceptíveis em sua amplitude.

\section{Perspectivas metodológicas}

\subsection{Método quantitativo}

Segundo Minayo (2005, p. 71), a abordagem quantitativa está relacionada a uma abordagem dedutiva, que consiste em, a partir de uma teoria conhecida ou de lei geral, observar casos particulares procurando confirmar a hipótese investigada ou gerar outras, ou seja, busca verificar resultados por meio de objetivos previamente definidos.

Ainda segundo a autora, para predizer e controlar eventos, comportamentos e outros desfechos, o pesquisador deve quantificar as causas e os efeitos, e isolá-los de seu contexto, a fim de tornar o processo, segundo a visão positivista, o mais objetivo possível.

A abordagem quantitativa pode ser resumida nos seguintes princípios: atua dentro da visão positivista; usa métodos estatísticos; trabalha com mensuração controlada; tem uma perspectiva externa aos dados; enfatiza dados confiáveis e aplicáveis; e considera a possibilidade de uma realidade estática.

Mullen e Iverson (1982, apud Minayo, 2005) destacam a importância da abordagem quantitativa para apresentar resultados que podem ser contados e expressos em números, taxas, proporções. Segundo os autores, esse tipo de abordagem é importante também para conhecer a cobertura, a concentração e a eficiência de programas, ações e intervenções, para avaliar objetivos bastante específicos, bem como para avaliar as diferenças de grau de um objeto que exige uma lógica de mais ou de menos. Além disso, a abordagem quantitativa estabelece relações significativas entre variáveis.

Segundo Fleury et. al. (1997, p. 32), a corrente quantitativa é freqüentemente relacionada ao conhecimento científico, entretanto, a compreensão de que o fenômeno social é ordenado e pode ser generalizado está presente em quase todas as abordagens metodológicas.

Ainda segundo a autora, os métodos quantitativos permitem, melhor que os outros, recolher, em um conjunto de elementos, informações comparáveis de um elemento a outro, ou seja, a condição necessária para a aplicação dos métodos quantitativos é, pois, que a observação repouse sobre um conjunto de elementos comparáveis.

A autora conclui que, em linhas gerais, pode-se afirmar que as abordagens quantitativas apresentam vantagens em termos de versatilidade, velocidade, custo, objetividade e processo, entretanto, alerta para o fato de algumas pesquisas não serem tão cuidadosas nos procedimentos, o que pode levar a resultados que não são válidos nem confiáveis.

\subsection{Método qualitativo}

Para Deslandes e Gomes (2004, p. 84), a abordagem qualitativa, embora tenha como foco central a compreensão do significado das ações sociais, ancora-se em diferentes bases disciplinares, metodológicas e paradigmáticas, formando um conjunto de atividades interpretativas.

A preferência por este tipo de abordagem é bastante recente, tendo se desenvolvido nos últimos 20 anos e, segundo Minayo (2005, p. 82) o método qualitativo trabalha com atitudes, crenças, comportamentos e ações, procurandose entender a forma como as pessoas interpretam e conferem sentido a suas experiências e ao mundo em que vivem. Em síntese, o objetivo é a compreensão da relação entre o mundo real e a subjetividade.

Miles e Huberman (1994, apud Fleury et al. 1997 b, p. 285) resumem as vantagens do método qualitativo nos seguintes pontos:

- A influência do contexto não é desprezada, mas levada em conta uma vez que os dados são coletados junto da situação específica, focada e vinculada a um contexto; a possibilidade de compreensão de temas latentes, subjacentes e não óbvios é muito grande;

- são de grande potencial para revelar a complexidade do fenômeno social;

- os dados coletados são menos sujeitos ao controle racional dos membros da organização, logo tendem a ser menos contaminados pelos interesses políticos e pelos mecanismos de defesa e recalque; 
- como os dados enfatizam as situações reais das pessoas, são adequados para identificar os significados que elas atribuem aos eventos, processos, pressupostos e os conectar ao mundo real;

- como os dados são coletados no eixo do tempo, são poderosos para estudar qualquer processo, ou seja, como e porque as coisas acontecem e mesmo avaliar a causalidade:

- permitem também visualizar mais claramente possibilidades de mudança, bem como os focos de resistência;

- são úteis, por fim, para suplementar, validar, explicar, iluminar ou reinterpretar dados quantitativos.

Por outro lado, os mesmos autores reconhecem que os dados qualitativos têm problemas e pontos fracos, que podem ser resumidos na seguinte conformidade:

- a coleta de dados demanda muito trabalho e tempo;

- possibilidade de viés do pessoal de campo;

- dificuldades com os métodos de analise;

- problemas de validade e fidedignidade.

Com relação à análise dos dados qualitativos, apontam-se os seguintes problemas:

- definição de um quadro de referência para propiciar clareza e foco para o trabalho de campo;

- bases de dados: o problema crítico é o tempo. O modo mais usual de gravar dados qualitativos é através de anotações que precisam ser convertidas em relatórios e a quanto mais estes demoram para ser redigidos, pior se torna a qualidade: muito é esquecido, simplificado ou reinterpretado à luz de eventos mais recentes.

- Análise dos dados: o primeiro passo está relacionado com a 'redução dos dados' - que é a forma preliminar de análise que refina e revisa o quadro de referência, sugere novos caminhos para coleta de dados e torna os dados disponíveis para montagem final em estudos de casos ou análise comparativa. Isto sugere alguma forma de pré-análise; entretanto, a questão da validade e fidedignidade da aplicação das categorias pelos pesquisadores é crítica.

Fleury et. al. (1997b p. 286) concluem que a opção por uma abordagem qualitativa apresenta vantagens e limitações específicas, que devem ser consideradas em função dos objetivos da pesquisa a ser realizada. Entretanto, a coerência entre a concepção do objeto a ser estudado e a abordagem metodológica a ser adotada é um aspecto que deve prevalecer sobre eventuais interesses circunstanciais.

\subsection{Aportes entre os métodos}

O estudo descritivo pode abordar aspectos amplos de uma sociedade, levantamentos de opinião e atitudes da população, caracterização do funcionamento de organizações, identificação de comportamento de um grupo ou de grupos. Os estudos que procuram investigar a correlação entre variáveis são fundamentais para as diversas ciências sociais, porque permitem controlar, simultaneamente, grande número de variáveis por meio de técnicas estatísticas de correlação, especificar o grau pelo qual diferentes variáveis estão relacionadas, oferecendo ao pesquisador entendimento do modo pelo qual as variáveis estão operando.

O método qualitativo difere, em principio, do quantitativo à medida que não emprega um instrumental estatístico como base do processo de análise de um problema, uma vez que não pretende numerar ou medir unidades.

O aspecto qualitativo de uma investigação pode estar presente até mesmo nas informações colhidas por estudos essencialmente quantitativos, não obstante perderem seu caráter qualitativo quando são transformadas em dados quantificáveis, na tentativa de se assegurar a exatidão no plano dos resultados.

Uma modalidade de transformar dados qualitativos em elementos quantificáveis, bastante empregada por pesquisadores, consiste em utilizar como parâmetros o emprego de critérios, categorias, escala de atitudes ou, ainda, identificar com que intensidade, ou grau, um conceito, uma atitude, uma opinião se manifesta.

Richardson et al. (1999, p. 88) consideram que embora existam diferenças ideológicas profundas, três instancias de integração entre ambos métodos (quantitativo e qualitativo) podem ser identificadas: no planejamento da pesquisa, na coleta de dados e na análise da informação.

Ainda de acordo com Richardson et al (1999, p. 88), pode-se perceber aportes do método qualitativo no quantitativo, e vice-versa. A saber:

\section{Aporte do método qualitativo ao quantitativo}

No planejamento da pesquisa, a discussão com o grupo que participará da investigação, o uso de entrevistas e a observação podem melhorar a formulação do problema, o levantamento de hipóteses e a determinação de amostras. 
$\mathrm{Na}$ coleta de dados, entrevistas, observações e discussões em grupo podem enriquecer as informações obtidas, particularmente pela profundidade e pelo detalhamento das técnicas qualitativas.

$\mathrm{Na}$ análise da informação, as técnicas qualitativas permitem verificar os resultados dos questionários e ampliar as relações descobertas.

\section{Aporte do método quantitativo ao qualitativo}

No planejamento da pesquisa, a utilização de um questionário prévio no momento da observação ou entrevista pode contribuir para delimitar o problema estudado e a informação coletada, permitindo identificar casos representativos ou não representativos em nível grupal ou individual.

$\mathrm{Na}$ coleta de dados o questionário prévio pode ajudar a evitar perguntas rotineiras e a identificar características objetivas, como, por exemplo, geopolíticas de uma comunidade, que podem influir no contexto da pesquisa.

\subsection{Triangulação}

As pesquisas sobre o comportamento das pessoas na utilização das Tecnologias de Informação e Comunicação (TIC's) nas organizações para a gestão da informação vêm sendo criticadas por predominarem pontos de vista positivistas e pelo uso intenso de metodologias quantitativas. Alguns autores têm procurado alternativas metodológicas que ajudem a dar conta de dimensões normalmente esquecidas, mas essenciais para compreender a implementação, uso e impactos das TIC's.

A dificuldade em relação aos estudos voltados à área de gestão da informação, quando se pretende estudar os comportamentos e as atitudes profissionais das pessoas envolvidas no processo, reside no fato de se deparar com elementos subjetivos e complexos, surgindo, assim, a necessidade do aprofundamento de técnicas e métodos que venham a satisfazer uma necessidade mais ampla de investigação.

Para ser considerada triangulação a pesquisa deve satisfazer as seguintes condições: deve ter pelo menos um método qualitativo de coleta de dados; deve ter pelo menos um método quantitativo de coleta de dados; os dados qualitativos e quantitativos devem ambos estar presentes e terem sido ambos analisados; a pesquisa deve endereçar-se a uma questão teórica. Juntando múltiplos métodos, como a observação, entrevistas e gravações vão levar a uma construção de realidades mais válida, de confiança e diversificada.

Para melhorar a análise e entendimento da construção de outros, a triangulação é um passo feito pelos pesquisadores para envolver várias interpretações de dados em diferentes tem- pos e lugares de investigadores ou pares de pesquisadores.

Neste sentido, a perspectiva de utilização da metodologia da triangulação busca agregar vários métodos, como objetivo de se obter uma melhor percepção de eventuais fenômenos que normalmente não são percebidos pelos métodos existentes aplicados de forma isolada, uma vez que o pesquisador, com a metodologia citada, pode utilizar várias ferramentas que visam a coletar sejam dados objetivos (quantitativos), sejam subjetivos (qualitativos), de forma que obtenha ao final da coleta, um panorama completo das vivências cotidianas no ambiente informacional das organizações.

Segundo Fleury et. al. (1997b p. 288), vários autores utilizam a combinação dos métodos qualitativos e quantitativos no estudo de um mesmo fenômeno, bem como alguns autores sugerem a combinação de técnicas quantitativas como questionários e análise de arquivos com técnicas qualitativas como entrevista e observação. A eficácia do método da triangulação é fundamentada na premissa de que a fraqueza de um único método será compensada pela força de outro (Duncan, 1986, apud Fleury et al., 1997).

Gallivan (1997, p. 420) utiliza o termo triangulação para "a pesquisa que integra trabalho de campo e levantamento quantitativo (survey) para responder uma questão empírica". Para ser considerada triangulação a pesquisa deve satisfazer as seguintes condições: deve ter pelo menos um método qualitativo de coleta de dados; deve ter pelo menos um método quantitativo de coleta de dados; os dados qualitativos e quantitativos devem ambos estar presentes e terem sido ambos analisados; a pesquisa deve endereçar-se a uma questão teórica.

Assim, conduzir algumas entrevistas para elaborar um questionário não é fazer triangulação, pois as entrevistas normalmente não são apresentadas nas análises. Pesquisas que colhem dados brutos sem nenhuma análise quantitativa, também, estão excluídas. Na triangulação deve haver necessariamente a integração entre os dois métodos de análise.

O uso de métodos mistos depende do problema de pesquisa. No estudo dos impactos das novas tecnologias, por exemplo, o uso da triangulação pode ser vantajoso porque permite obter dados no plano individual e, também, no plano organizacional, facilitando que esses dois níveis de análise sejam integrados (Gallivan, 1997, p. 423). 
Segundo Golafshani (2003, p. 603), a triangulação é tipicamente uma estratégia para melhorar a validade e a confiabilidade da pesquisa. Patton (2002, apud Golafshani, 2003, p. 603) defende o uso da triangulação indicando "a triangulação como um estudo de combinação de métodos. Isso pode significar estudo de vários tipos de métodos ou dados, incluindo o uso tanto de uma abordagem quantitativa quanto qualitativa".

Segundo Jick (1979, apud Fleury et. al., 1997, p. 288), existem quatro tipos básicos de triangulação existentes:

- Triangulação de dados, que tem 3 subtipos: (a) tempo, (b) espaço e (c) pessoa. A análise das pessoas, por sua vez tem três tipos subtipos: (i) agregado, (ii) interativo, e (iii) coletividade.

- Triangulação do investigador, que consiste em utilizar múltiplos observadores de um mesmo objeto ao invés de um único.

- Triangulação de teoria, que consiste em utilizar múltiplas perspectivas ao invés de uma única ao mesmo conjunto de objetos.

- Triangulação metodológica, da qual podemos destacar dois tipos de triangulação: (a) o chamado entre métodos, quando dois ou mais métodos distintos são congruentes e desenvolvem dados comparáveis; e (b) o intramétodo, que utiliza múltiplas técnicas dentro de um dado método para coletar e interpretar dados. Em resumo, triangulação 'intramétodo' essencialmente envolve verificação cruzada de consistência interna e confiabilidade, ao passo que a triangulação 'entre métodos' testa o grau de validade externa.

Dessa forma, sugere-se, para o estudo da gestão da informação em ambientes informacionais das organizações, com o foco nas pessoas, a triangulação metodológica, tanto na sua forma entre métodos (quantitativo e qualitativo), tanto quanto na sua forma intramétodos, na qual está previsto o uso de múltiplas técnicas de coleta de dados: questionário, entrevistas, análise de documentos e observação. Optou-se por esta alternativa, uma vez que se busca tanto a validação externa (entre métodos) quanto a consistência interna e confiabilidade (intramétodo).

Para Creswell \& Miller (2000, p. 126, apud Golafshani, 2003, p. 604), a triangulação pode ser definida como um processo de validação, no qual os pesquisadores buscam por convergências entre múltiplas e variadas fontes de informação para formar temas ou categorias de estudo, de forma a se obter dados mais confiáveis.

\section{Considerações finais}

Esta discussão buscou destacar a questão da complexidade dos estudos voltados à área de gestão da informação, se o pesquisador quiser adotar a perspectiva de estudar as pessoas envolvidas no processo.

Esta dificuldade surge do afloramento de elementos subjetivos, característicos dos seres humanos, e que, muitas vezes, não são totalmente explícitos, e nem sempre podem ser apreendidos apenas pelo uso de métodos quantitativos.

Assim, surge a necessidade de adoção de técnicas e métodos que visem a uma investigação mais ampla e aprofundada, tanto do ponto de vista da coleta como da interpretação dos dados.

Quando se sugere o método da triangulação, seja na sua variedade entre métodos, seja na intramétodos, o que se busca é agregar vários métodos, ou técnicas dentro de um método, na busca de uma melhor percepção de eventuais fenômenos que não são percebidos pelos métodos existentes, se forem aplicados de forma isolada, mas que podem revelar vários aspetos se aplicados de forma conjunta ou coordenada.

Desta forma, demonstra-se a existência de um campo de estudo a ser explorado e desenvolvido nos ambientes informacionais das organizações, o da questão metodológica, contribuindo, assim, para o desenvolvimento de pesquisas voltadas para a gestão da informação.

\section{Referências}

Castells, M. (2001). A sociedade em rede: a era da informação - economia, sociedade e cultura. Rio de Janeiro: Paz e Terra, 2001.

Coriat, B. (1988). Automação programável: novas formas e conceitos de organização da produção. // Carvalho, R.; Schmitiz, H. (Org.). Automação, competitividade e trabalho. São Paulo: Hucitec, 1988. 13-61.

Creswell, J. W.; Miller, D. L. (2000). Determining validity in qualitative inquiry. Theory into Practice, 39:3 (2000) 124131.

Davenport, T.; Prusak, L. (2001). Ecologia da informação: por que só a tecnologia não basta para o sucesso na era da informação. São Paulo: Futura, 2001.

Deslandes, S.F., Gomes, R. (2004). A pesquisa qualitativa nos serviços de saúde: notas teóricas. // Bosi, M.L., Mercado, F.J. (orgs.). Pesquisa qualitativa de serviços de saúde. Petrópolis: Vozes, 2004.

Duncan, W.J. (1986). A proposal for a multimethod approach to organizational culture research. Birmingham: University of Alabama, 1986.

Fleury, M.T.L.; Shinyashiki, G.; Stevanato, L.A. (1997). Entre a antropologia e a psicanálise: dilemas metodológicos dos estudos sobre cultura organizacional. // Revista de Administração. 32:1 (janeiro/março, 1997) 23-37. 
Fleury, M. T. L.; Shinyashiki, G. T.; Stevanato, L. A. (1997b). Arqueologia teórica e dilemas metodológicos dos estudos sobre a cultura organizacional. // Motta, F. C. P.; Caldas, M. P. Cultura organizacional e cultura brasileira. São Paulo: Atlas, 1997b.

Fleury, M. T. L.; Silva, S. M. da. (2003). Cultura organizacional e tecnologia de informação: um estudo de caso em organizações universitárias. // Ruben, G.; Wainer, J.; Dwyer, T. (Orgs.). Informática, organizações e sociedade no Brasil. São Paulo: Cortez, 2003. 161-186.

Gallivan, M. J. (1997). Value in triangulation: a comparison of two approaches for combining qualitative and quantitative methods. // Lee, A.S; Liebenau, J; DeGross, J.I. (Eds.). Information systems and qualitative research. Philadelphia: FIP, 1997.

Golafshani, N. Understanding reliability and validity in qualitative research. The Qualitative Report. 8:4 (2004) 597606. http://www.nova.edu/ssss/QR/QR8-4/golafshani. pdf. (30 jan. 2008).

Harvey, D. (1992). A condição pós-moderna. São Paulo: Loyola, 1992.

Jick, T. D. (1979). Mixing qualitative and quantitative methods: triangulation in action. // Administrative Science Quaterly. 24 (1979) 602-611.

Lojkine, J. (1995). A revolução industrial. São Paulo: Cortez, 1995.

Martin, J. (1991). Reengenharia da informação. Rio de Janeiro: Campus, 1991

Masi, D. (1999). A sociedade pós-industrial. São Paulo: SENAC, 1999.

Miles, M.B., Huberman, A.M. (1994). Qualitative data analysys. Thousand Oaks: Sage Publications, 1994.

Minayo, M.C.S., Assis, S.G., Souza, E.R. (2005). Avaliação por triangulação de métodos. Rio de Janeiro: Editora Fiocruz, 2005.

Moraes, C. R. B; Fadel, B. (2006). Ambiência organizacional, gestão da informação e tecnologia. // Valentim, M. L. P. (Org.). Informação, conhecimento e inteligência organizacional. Marília: Fundepe Editora, 2006.

Mullen, P. D., Iverson, D. Qualitative methods for evaluative research in health education programs. // Health Education. 13: 3 (1982) 11-18.

Patton, M. Q. (2002). Qualitative evaluation and research methods. Thousand Oaks (CA): Sage Publications, 2002.

Pochmann, M. (2003). As possibilidades do trabalho e a nova economia no Brasil. // Ruben, G.; Wainer, J.; Dwyer, T. (Org.). Informática, organizações e sociedade no Brasil. São Paulo: Cortez, 2003. 93-129.

Ponjuán Dante, G. (2007). Gestión de información: dimensiones e implementación para el éxito organizacional. Gijón: Trea, 2007.

Richardson, R. J. et al. (1999). Pesquisa social: métodos e técnicas. São Paulo: Atlas, 1999.

Schaff, A. (1995). A sociedade informática. São Paulo: Brasiliense. 1995.

Valentim, M. L. P. Inteligência competitiva em organizações: dado, informação e conhecimento. DataGramaZero, 3:4 (ago. 2002). http://www.dgz.org.br/ago02/Art_02.htm. (22 de maio de 2008). 